\title{
Mutations in maltose-binding protein that alter affinity and solubility properties
}

\author{
Iris H. Walker • Pei-chung Hsieh • Paul D. Riggs
}

Received: 24 February 2010 /Revised: 18 May 2010 /Accepted: 24 May 2010 / Published online: 10 June 2010

(C) The Author(s) 2010. This article is published with open access at Springerlink.com

\begin{abstract}
Maltose-binding protein (MBP) from Escherichia coli has been shown to be a good substrate for protein engineering leading to altered binding (Marvin and Hellinga, Proc Natl Acad Sci U S A 98:4955-4960, 2001a) and increased affinity (Marvin and Hellinga, Nat Struct Biol 8:795-798, 2001b; Telmer and Shilton, J Biol Chem 278:34555-34567, 2003). It is also used in recombinant protein expression as both an affinity tag and a solubility tag. We isolated mutations in MBP that enhance binding to maltodextrins 1.3 to 15 -fold, using random mutagenesis followed by screening for enhanced yield in a microplatebased affinity purification. We tested the mutations for their ability to enhance the yield of a fusion protein that binds poorly to immobilized amylose and their ability to enhance the solubility of one or more aggregation-prone recombinant proteins. We also measured dissociation constants of the mutant MBPs that retain the solubility-enhancing properties of MBP and combined two of the mutations to produce an MBP with a dissociation constant 10-fold tighter than wildtype MBP. Some of the mutations we obtained can be rationalized based on the previous work, while others indicate new ways in which the function of MBP can be modified.
\end{abstract}

Keywords Maltose-binding protein $\cdot$ Periplasmic binding proteins · Altered affinity · Mutational analysis .

Recombinant fusion proteins $\cdot$ Solubility enhancement

Electronic supplementary material The online version of this article (doi:10.1007/s00253-010-2696-y) contains supplementary material, which is available to authorized users.

I. H. Walker · P.-c. Hsieh • P. D. Riggs $(\bowtie)$

New England Biolabs,

240 County Rd,

Ipswich, MA 01938-2723, USA

e-mail: riggs@neb.com
The Escherichia coli maltodextrin-binding protein (MBP) is a member of the periplasmic binding protein family and functions in the transport of maltodextrins. The role of MBP is to bind maltodextrins at the outer membrane porin LamB and release them to the MalEFK transport apparatus in the inner membrane. A high affinity is required when in position at the outer membrane to bind maltodextrins, but the sugar must be released at the inner membrane when it docks with the transport apparatus. It accomplishes this in part by having two conformations, the closed form which favors binding and the open form which favors release. In addition, MBP acts as a sensor for maltodextrins in the chemotactic system, interacting with the signal transduction protein Tar to regulate motility. The affinity characteristics of MBP for maltodextrins in vivo have been selected to optimize these functions.

MBP is also a useful tag for the expression and purification of recombinant proteins in three ways: improving translational expression, providing an affinity purification strategy, and enhancing the solubility of many refractory proteins. Microbial expression systems such as those developed for E. coli are often the first choice for preparing large amounts of recombinant proteins due to their low cost and high yield. To facilitate the expression and purification of a target protein, one method that is in common use is to fuse a tag to the protein. A good tag has properties that facilitate highlevel translational expression when fused to the target protein, as well as providing a simple one-step affinity purification that allows the target protein to be purified from the expression milieu. MBP is commonly used as a tag for expression and purification of foreign proteins produced in E. coli. Fusion of the $\mathrm{C}$ terminus of MBP to the $\mathrm{N}$ terminus of a target protein facilitates the expression of the fusion protein in E. coli. MBP and MBP fusions can be purified in a single step by binding to a chromatography matrix containing 
any of a number of glucose- $\alpha 1 \rightarrow 4$-glucose polysaccharides such as amylose, starch or other maltodextrins. MBP is also useful as a "solubility tag." Proteins that are soluble in their native host are sometimes insoluble when expressed in E. coli. For many of these proteins, fusion to MBP renders them soluble (Kapust and Waugh 1999).

MBP has previously been engineered for changes in binding affinity and specificity. The Kd of MBP for maltose and maltotriose in vitro is about 2 and $0.4 \mu \mathrm{M}$, respectively (Miller et al. 1983; Telmer and Shilton 2003). The X-ray crystal structure of MBP shows that MBP consists of two domains, with a cleft between the domains where the polysaccharide binds (Duan and Quiocho 2002; Sharff et al. 1992; Spurlino et al. 1991). The domain that contains the $\mathrm{N}$ terminus is named domain $\mathrm{I}$, and the domain that contains the $\mathrm{C}$ terminus is named domain II. Three loops cross between the two domains to form a hinge. Researchers have used the structure of MBP to make directed mutations in order to alter the binding properties of MBP. Using directed mutagenesis based on the structure, MBP has been engineered to bind zinc (Marvin and Hellinga 2001a). In addition, two reports have described using the structure to make directed mutations to the region behind the hinge that increases the affinity of MBP for maltose and maltotriose (Marvin and Hellinga 2001b; Telmer and Shilton 2003). However, the mutations made using this approach can decrease MBP's ability to enhance the solubility of proteins to which it is fused, thus reducing its utility as a tag (Nallamsetty and Waugh 2007).

In this study, we explored the possibility that there might exist mutations in MBP that alter its conformation and/or its affinity for maltodextrins without compromising its solubilityenhancing properties, either by subtle changes in the hydrophobic core of the protein or by surface changes that do not compromise its folding or solubility. We set out to identify such mutations by random mutagenesis and screening for mutants that out-performed wild-type MBP2 in an affinity purification.

\section{Materials and methods}

Materials Restriction enzymes, $\beta$-agarase, DNA polymerases, T4 ligase, antarctic phosphatase, Litmus 38, the pMAL Protein Fusion and Purification System including pMAL-c2X, pMAL-c2G, and pMAL-c4X, the USER Friendly Cloning kit, amylose resin, anti-MBP monoclonal antibody linked to horseradish peroxidase, and synthetic oligonucleotides were obtained from New England Biolabs. The nuclease Benzonase from Serratia marcescens was purified as an MBP fusion protein and separated from MBP by digestion with factor Xa protease, using the pMAL system (data not shown). Whatman Unifilter 800 microplates with filter bottoms and Immulon $2 \mathrm{HB}$ microplates were purchased from VWR. The MinElute DNA Extraction and QIAquick Spin kits were purchased from Qiagen. The detergent MEGA 10 was purchased from Dojindo. Hen egg white lysozyme and Coomassie brilliant blue $\mathrm{R}$ were purchased from Sigma-Aldrich. Disposable polypropylene columns (\#732-6008) were purchased from BioRad; 10 $20 \%$ gradient gels were purchased from either Daiichi or Invitrogen/Novex.

Strains and plasmids Host strains TB1, ER1992, ER2502, ER2984, NEB 5-alpha, NEB Turbo, and NEB Express were obtained from New England Biolabs. The source of the Bacillus circulans chitin binding domain (CBD) was pMB50, kindly provided by M. Xu (see Supplemental data). The construction of the pMAL derivatives pSN1578 and pIH1684 is described in the supplemental data. The pMAL-DHFR and pMAL-GAPDH plasmids were constructed using a protocol similar to ligation-independent cloning (Aslanidis and de Jong 1990), except in this case, the overhangs created were short and still require ligase to produce a transformable plasmid (see Supplemental data). The DHFR and GAPDH regions from the resulting plasmids were then transferred into other pMAL derivatives as AvaI, SbfI or AvaI, HindIII fragments.

PCR mutagenesis Random PCR mutagenesis was carried out as described in (Fromant et al. 1995). Briefly, four reactions were carried out, each with three of the four nucleotides at a concentration of $0.2 \mathrm{mM}$ and the fourth nucleotide at $0.5 \mathrm{mM}$. PCR was performed using Taq polymerase in the presence of $6 \mathrm{mM} \mathrm{MgSO}_{4}$. For the first library, the malE gene was amplified with the primers $5^{\prime}$ GGAGACAUGAATTCAATGAAAATCGAAGAA and 5' GGGAAAGUAAGCTTAATCCTTCCCTCGATC, using pMAL-c2X as a template. PCR fragments were cloned into linearized pNEB208A using the USER Friendly Cloning Kit, following the manufacturer's instructions. For the second library, the malE gene was amplified with the primers 5'CACGAGCAATTGACCAACAAGGAC and 5'GATCGAGAGCTCGAATTAGTCTGC. Both the PCR product and pIH1684 were cut with MfeI and SacI and gel purified, and the two fragments were ligated. Transformants from each library were grown overnight in $0.9 \mathrm{~mL} \mathrm{LB}+10$ $\mathrm{uM}$ IPTG and $100 \mathrm{ug} / \mathrm{mL}$ ampicillin and then lysed by adding $0.1 \mathrm{~mL}$ of a detergent/lysozyme/nuclease solution,

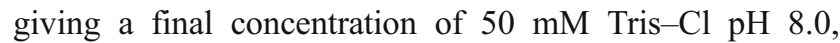
$50 \mathrm{mM} \mathrm{NaCl}, 0.2 \mathrm{mM}$ EDTA, $0.2 \mathrm{mg} / \mathrm{mL}$ lysozyme, $0.3 \%$ MEGA-10, and $20 \mathrm{U} / \mathrm{mL}$ of Benzonase (to reduce viscosity; modified Kunitz units (Friedhoff et al. 1994)) and incubating for $2 \mathrm{~min}$ at room temperature.

Screening MBP mutants by affinity purification Of the extracts prepared, $0.7 \mathrm{~mL}$, as described above, was applied 
to $100 \mathrm{uL}$ of amylose resin in a well of a Unifilter 800 microplate, and each well was washed with $2 \mathrm{~mL}$ of $20 \mathrm{mM}$ Tris-Cl, $0.2 \mathrm{M} \mathrm{NaCl}, 1 \mathrm{mM}$ EDTA, pH 7.4 (column buffer, $\mathrm{CB}$ ) containing $0.2 \%$ Tween 20 , then with $2 \mathrm{~mL}$ of $\mathrm{CB}$ without Tween 20 , and finally with $1 \mathrm{~mL}$ of $10 \mathrm{mM}$ sodium phosphate, $0.2 \mathrm{M} \mathrm{NaCl}, 1 \mathrm{mM}$ EDTA, $\mathrm{pH}$ 7.2. The protein bound to the amylose resin was then eluted with $0.2 \mathrm{~mL}$ of $10 \mathrm{mM}$ maltose, $10 \mathrm{mM}$ sodium phosphate, $0.2 \mathrm{M} \mathrm{NaCl}$, 1 mM EDTA, $\mathrm{pH}$ 7.2.

The eluate was transferred to an Immulon $2 \mathrm{HB}$ microplate and incubated overnight at $4{ }^{\circ} \mathrm{C}$. The microplate wells were then emptied, washed twice with $20 \mathrm{mM}$ Tris-Cl, $150 \mathrm{mM}$ $\mathrm{NaCl}, \mathrm{pH} 7.5$ (TBST), then blocked with $0.36 \mathrm{~mL}$ TBST $+3 \%$ bovine serum albumin for $1 \mathrm{~h}$ at $37^{\circ} \mathrm{C}$. The wells were washed twice with TBST, then $0.2 \mathrm{~mL}$ of a 1:2,000 dilution of antiMBP monoclonal antibody linked to horse radish peroxidase in TBST $+3 \%$ bovine serum albumin was added to each well and the plate incubated at $37^{\circ} \mathrm{C}$ for $1 \mathrm{~h}$. The wells were emptied and then washed three times with TBST. The wells were developed with $0.01 \%$ o-phenylenediamine, $0.003 \%$ hydrogen peroxide in water. The detection reaction was stopped by adding $0.025 \mathrm{~mL} 4 \mathrm{M} \mathrm{H}_{2} \mathrm{SO}_{4}$, and wells were assayed spectrophotometrically at $490 \mathrm{~nm}$. Cells were recovered from samples corresponding to lysates that showed higher binding and elution as compared to wildtype MBP. These candidates were grown and retested to confirm the higher binding and elution. A list of the mutations is given in Table S1.

Subcloning and separation of mutations For the first library, the genes for candidate MBP mutants were processed with PCR from pNEB208A using the primers 5' GACTCATAT GAAAATCGAAGAAGGTAAACTGGTAATCTGGAT TAACGGC and 5' ATATAAGCTTTCACCTTCCCTC GATCCCGAGGT. The PCR fragment was cut with NdeI and HindIII and ligated into the pMAL derivative pIH1684 cut with the same enzymes. The second library was constructed directly in pIH1684, so testing proceeded without needing to subclone. Of the 25 mutants obtained in the screen, ten had silent mutations in addition to one or more missense mutations; the silent mutations were ignored for this analysis. Five were identical in sequence to another mutant, presumed to be siblings, and dropped. Nine had a single missense mutation. The remaining 11 had from two to five missense mutations. The mutations in the multiple mutants were reconstructed separately by either subcloning, when appropriate sites were available, or four primer mutagenesis as described in Guan and Kumar 2005. The PCR fragments produced were ligated into pIH1684.

Small-scale affinity purification of MBP and MBP fusions A 200-mL culture of each variant of MBP2 to be tested was grown in $\mathrm{LB}+0.1 \%$ glucose and $100 \mu \mathrm{g} / \mathrm{mL}$ ampicillin to early $\log$ phase, then induced by addition of IPTG to a final concentration of $0.3 \mathrm{mM}$. The cells were harvested after $2 \mathrm{~h}$ and resuspended in $5 \mathrm{~mL} \mathrm{CB}$, sonicated, and clarified at $9,000 \times \mathrm{g}$ for $30 \mathrm{~min}$. A $1.6-\mathrm{mL}$ sample of the supernatant was diluted to $8 \mathrm{~mL}$ with $\mathrm{CB}$ and applied to a $1-\mathrm{mL}$ amylose column. The column was washed with $16 \mathrm{~mL} \mathrm{CB}$ and eluted with $4 \mathrm{~mL}$ of $\mathrm{CB}+10 \mathrm{mM}$ maltose. The protein in the eluate was quantitated by spectrophotometry at $280 \mathrm{~nm}\left(\mathrm{~A}_{280}\right.$ of 1 corresponds to $\left.0.66 \mathrm{mg} / \mathrm{mL}\right)$. Values for wild-type (included in all experiments as a control) are the average of 19 experiments, and values for A312V (included as an additional control in most experiments) are the average of 16 experiments; all others are the average of 2-8 experiments and are expressed as \% yield relative to wild-type in the same experiment, then normalized to the overall wild-type average.

Solubility enhancement of MBP fusions A 10-mL culture of NEB Express containing the pMAL-DHFR or pMALGAPDH derivative was grown to $2 \times 10^{8}$ cells $/ \mathrm{mL}$ in $\mathrm{LB}+$ $0.1 \%$ glucose and $100 \mu \mathrm{g} / \mathrm{mL}$ ampicillin, induced with $0.3 \mathrm{mM}$ IPTG, incubated for an additional $2 \mathrm{~h}$, then cells were harvested by centrifugation at $3,000 \times g$ in a microfuge. Each pellet was resuspended in $1 \mathrm{~mL}$ of $\mathrm{CB}$ and lysed by sonication. The lysate was centrifuged for $2 \mathrm{~m}$ at $14,000 \times g$, and the supernatant was removed and designated the soluble fraction. The pellet was resupended in $1 \mathrm{~mL}$ of the same buffer and designated the insoluble fraction. A sample (4 uL) of each fraction was run on SDS-PAGE for each strain, the gels were dried and scanned, and the amount of MBP fusion protein in each lane was quantitated using ImageJ (Abramoff et al. 2004). Results are expressed as a ratio of soluble MBP fusion protein to total MBP fusion protein present in the soluble and insoluble lanes.

Determination of dissociation constants $\mathrm{Kd}$ measurements were made by measuring maltose-induced fluorescence quenching (Miller et al. 1983) using a Perkin-Elmer LS50B luminescence spectrophotometer. All measurements were carried out at room temperature $\left(25-26^{\circ} \mathrm{C}\right)$, at a protein concentration of $15 \mu \mathrm{g} / \mathrm{mL}(0.32 \mu \mathrm{M})$ and an excitation wavelength of $290 \mathrm{~nm}$. Emission scans were done with excitation slit width of $2.5 \mathrm{~nm}$ and an emission slit width of $5.0 \mathrm{~nm}$. Titrations with maltose or maltotriose were carried out with excitation and emission slit widths of $5.0 \mathrm{~nm}$, and an emission wavelength of 330 and/or $350 \mathrm{~nm}$, depending on the mutant (see "Results"). The data were plotted as percent quenched vs. input maltose/ maltotriose concentration, and the dissociation constant determined by a curve-fit using the equilibrium binding equation in Miller et al. 1983 and the software KaleidaGraph 4 (Synergy Software). 


\section{Results}

MBP mutations that affect affinity purification We undertook the present study to see if we could identify mutations in MBP that would increase its ability to bind and elute from an amylose affinity resin. We hoped that by screening random mutants for this property, we might identify mutations that affect the affinity without altering the properties that make MBP a useful affinity tag, most importantly its ability to enhance the solubility of proteins that tend to be insoluble when expressed in E. coli. Wildtype MBP of $E$. coli is made as a precursor with an Nterminal signal peptide and secreted into the periplasm where the signal peptide is removed. For this study, we used a cytoplasmic derivative of MBP called MBP2, which differs from the mature MBP protein in that it has a methionine at the $\mathrm{N}$ terminus in place of the signal peptide, and the last four residues are replaced by a 20-residueengineered linker and residues encoded by a MCS on the pMAL vectors. We used error-prone PCR to create mutant alleles of the gene that encodes MBP2 and screened about 4,000 isolates from two independent libraries of MBP2 mutants. Among the 19 mutations obtained, we identified substitutions at 14 positions in the amino acid sequence and one frameshift mutation. The frameshift was in the last base of the malE gene present in our construct and affected the residues that are encoded by the engineered linker. Many of the mutants contained multiple mutations. We separated the mutations and tested them individually to identify which of the mutations were responsible for the increase in yield from the affinity purification relative to MBP2 (high-yield phenotype). In all cases but two, we found that a single mutation could account for the high yield of the original mutant (data not shown). However, we cannot rule out that the additional mutations that have no phenotype when tested alone could contribute to the phenotype of the original mutant. In the two cases where more than one mutation contributed to the phenotype, we found other variants of MBP2 that contained just one of each of the changes. The locations of the mutations in the primary sequence are shown in Fig. 1, while Fig. 2 shows a cartoon of the structure of MBP and the location of the residues mutated. All but one of the mutations are located between residues 68 and 115 in the N-terminal half of the sequence and between residues 303 and 343 in the Cterminal half; the final mutation, the $\Delta 2682$ deletion, creates a frameshift which affects residues from 367 to the end. There is a hotspot for mutations in helix 14 of domain I, which contains a number of residues that interact with domain II in the open conformation (Marvin and Hellinga 2001a; Telmer and Shilton 2003).

Effect of mutations on affinity purification of $M B P 2$ and a fusion derivative After the initial identification of the mutations, we retested each MBP2 derivative in a $1-\mathrm{mL}$ column format. In order to test whether the increased yield of MBP2 would carry over to problematic fusion proteins, we also constructed pMAL vectors that would express an MBP2-chitin binding domain fusion (MBP2-CBD) for each of the mutations. Under the conditions we used, approximately $95 \%$ of the wild-type derivatives of both MBP2 and MBP2-CBD failed to bind or prematurely eluted from the amylose resin during the wash. The yield for wild-type MBP2 in these experiments was $2.3 \pm 0.85 \mathrm{mg} / \mathrm{L}$; the yield for wild-type MBP2-CBD was $3.7 \pm 1.1 \mathrm{mg} / \mathrm{L}$ (average of 19 experiments; error is the $95 \%$ confidence interval). All 19 mutations were tested as MBP2 and MBP2-CBD fusions, and the results are shown in Fig. 3a. The mutations increased the yield of MBP2 from 1.3- to 15-fold over wild-type MBP2. With the exception of V302L, Y307C, and $\mathrm{M} 321 \mathrm{~K}$, the mutations that led to an increase in MBP2 yield also led to an increase MBP2-CBD yield; unlike the V302L derivative, the V302A derivative of MBP2-CBD did give an increased yield.

Mutations that retain the solubility enhancement properties of MBP fusions In order to see if the mutations affected the ability of MBP2 to enhance the solubility of fused proteins, we constructed pMAL plasmids that expressed MBP2 fused to human dihydrofolate reductase (MBP2-DHFR) for each of the mutations. We chose DHFR from among the proteins whose solubility had previously been shown to be enhanced by MBP (Kapust and Waugh 1999). Figure 3b shows the results of this solubility assay. Five out of the 19 mutations retain all or most of the solubility enhancement property of MBP2 for this fusion: F67L, A312V, I317V, Q325R, and V343M. To test if the solubility-enhancement properties of these five mutations might extend to other proteins, we tested them as fusions to murine glyceraldehyde phosphate dehydrogenase (MBP-GAPDH), another protein whose solubility has been shown to be enhanced by MBP (Kapust and Waugh 1999). All of these mutations, with the possible exception of Q325R, were able to solubilize GAPDH about as well as wild-type MBP2 (Fig. 3b).

Combinations of mutations We made one class of double mutants to test whether the loss of solubility enhancement due to a change in one residue could be rescued by a second mutation, by testing the solubility of MBP2-DHFR derivatives with the $\mathrm{A} 312 \mathrm{~V}$ mutation along with substitutions at N323 and N332. The original mutations at these residues, N323Y and N332I, reduced the solubility of MBP2-DHFR, while $\mathrm{A} 312 \mathrm{~V}$ was neutral or slightly increased its solubility. We reasoned that another hydrophilic side chain as a substitute for asparagine would be more likely to retain solubility enhancement, and the addition of $\mathrm{A} 312 \mathrm{~V}$ might provide some additional increase in the solubility properties of the mutant. 
A1)

eccer (1)

$(A 2) \Rightarrow$

Cacer

MKIEEGKLVIWINGDKGYNGLAEVGKKFEKDTGIKVTVEHPDKLEEKFPQ

50

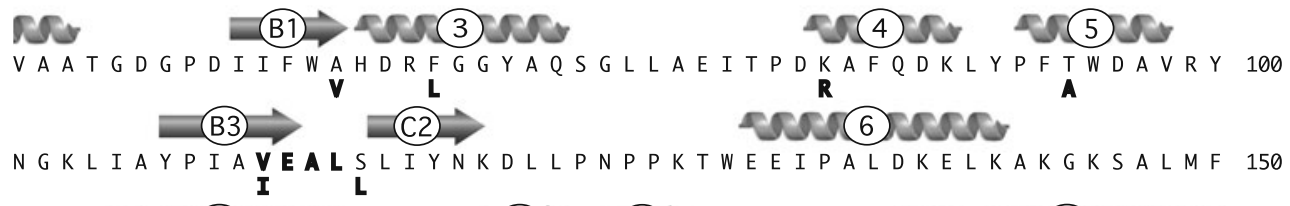

ecer 7)ecer

(D1) $\Rightarrow$ (D2) $\Rightarrow$

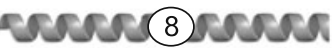

NL QEPYFTWPLIAADGGYAFKYENGKYDIKDVGVDNAGAKAGLTFLVDLI 200

cacigere $\Rightarrow$

K N K H M N A D T D Y S I A E A A F N K GE TA M T I N G P W A W S N I D T S K V N Y G VTVLPT 250

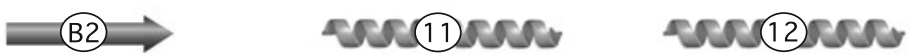

FKG QPSKPF V G V LSAGINAASPNKELAKEFLENYLLTDEGLEAVNKDKPL 300

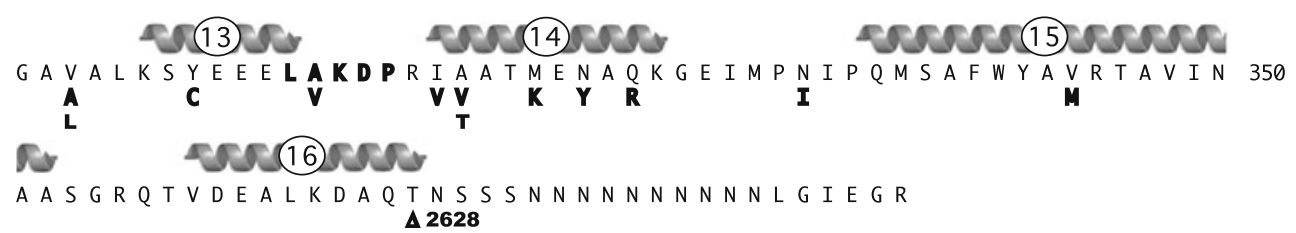

Fig. 1 Sequence of MBP2 and location of mutations. Mutations are indicated in bold under the amino acid sequence. $\Delta 2682$ indicates a deletion of a thymine at position 2682 in the DNA sequence, which leads to a frameshift of the subsequent residues in MBP2. Secondary structural elements are indicated above the sequence, arrows for $\beta$ sheets and helices for $\alpha$-helices, according to the PDB file 1ANF (Spurlino et al. 1991). The $\beta$-sheets are labeled with a letter to

indicate the structural element and a number to indicate the strand in that element; e.g., $\beta$-sheet "A" is made up of two strands, A1 and A2. Helices are indicated by a number. The numbering of the mutations we obtained ignores the N-terminal methionine present in MBP2, to simplify comparison to the structure and the previous literature. The sequences corresponding to the hinges between the two domains are indicated as bold letters embedded in the sequence
Fig. 2 MBP structure and location of mutated residues. Structure from PDB file 1ANF rendered using PyMOL.

Domain I is in green, domain II is in yellow, hinge regions are in cyan, and maltose carbons are in salmon. View is from the opposite side from the binding cleft. a Cartoon of MBP structure. b Location of mutated residues. Stereo view of the $\alpha$-carbon trace of MBP, with the residues where mutants were obtained in crimson
A

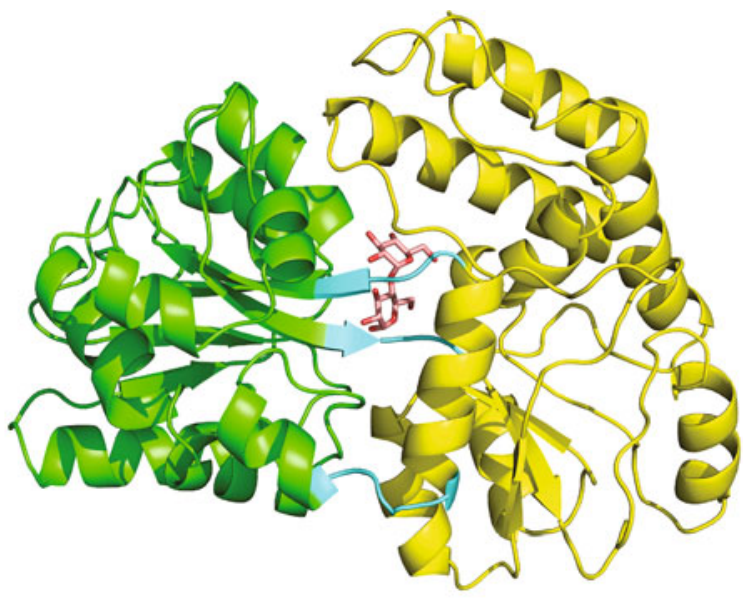

B

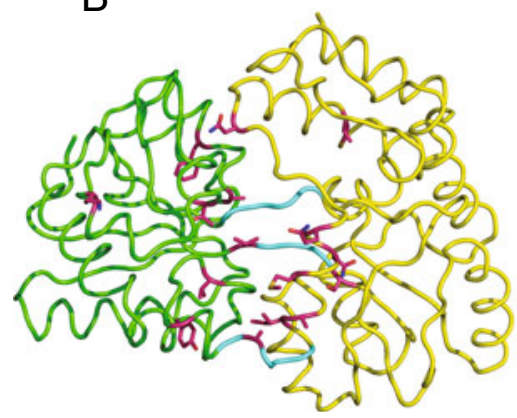

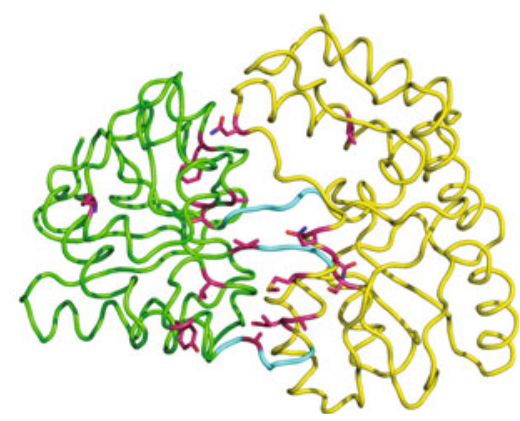


Fig. 3 a Yield of MBP2 and MBP2-CBD derivatives. MBP2, MBP2-CBD, and their mutant derivatives were purified on a 1 -mL amylose column as described in "Materials and methods" and the results graphed as the yield of protein relative to the yield of wild-type MBP2 in a purification performed in parallel. Values for wild-type MBP2 and $\mathrm{A} 312 \mathrm{~V}$ are the average of 19 experiments (included as controls in all experiments); values for the remaining mutants are the average of 2-8 experiments. Error bars are $95 \%$ confidence intervals. b Cells expressing MBP2-DHFR or MBP2-GAPDH and their mutant derivatives were lysed and fractionated into soluble and insoluble fractions, and the amount of soluble and insoluble fusion protein was quantitated, as described in "Materials and methods." Results are expressed as the ratio of soluble MBP fusion protein to total MBP fusion protein. Values for wild-type and $\mathrm{A} 312 \mathrm{~V}$ are the average of 19 and 16 experiments, respectively (included as controls in all or most experiments, respectively); all others are the average of three to four experiments and are expressed as $\%$ soluble relative to wild-type in the same experiment, then normalized to the overall wildtype average. Error bars are $95 \%$ confidence intervals

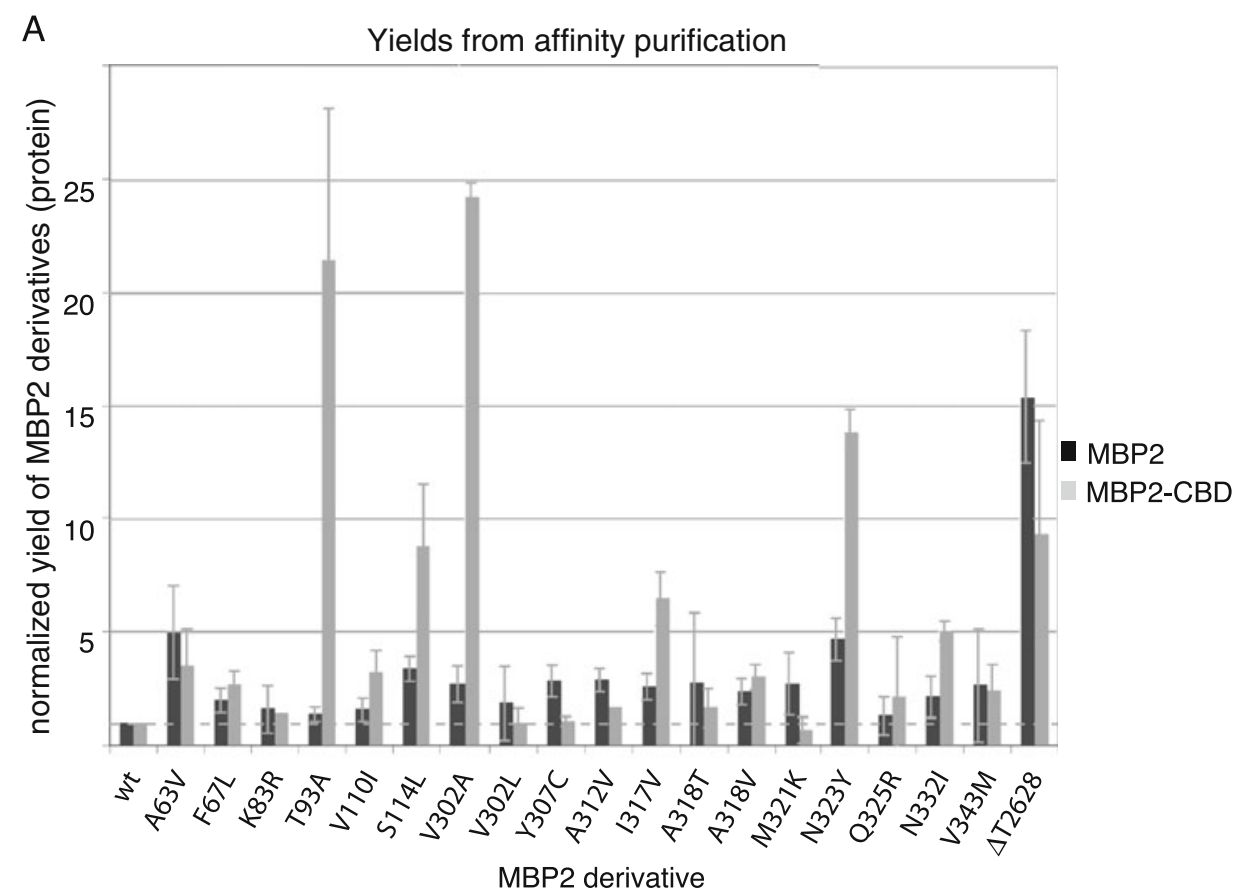

B Solubility of MBP2 fusion proteins

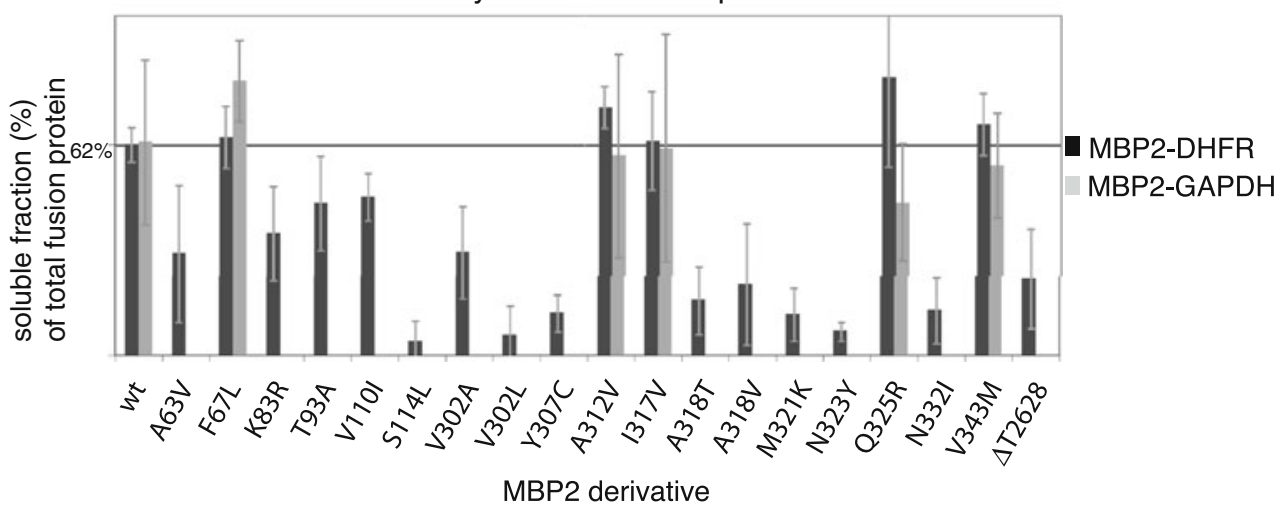

Thus, we tested the double mutants A312V N323Q, A312V N323R, A312V N332Q, and A312V N332R. All four of these mutants had a greatly reduced ability to solubilize the fused DHFR compared to wild-type MBP2, one fifth that of wildtype or less (data not shown).

We constructed another class of double mutants to see if two mutations that individually provided good solubility could be combined to produce a double mutant that retained the solubility property while also maintaining increased affinity. The double mutants we constructed were A312V I317V, A312V I317A, A312V Q325R, and A312V Q325K. The results are shown on Table 1. The A312V Q325X mutants showed reduced ability to enhance the solubility of DHFR compared to wild-type, but the A312V I317V and A312V I317A mutants had solubility properties at least equal to wild-type. Encouraged by these results, we tested the double mutants for their yield of MBP2 and MBP2$\mathrm{CBD}$ in the amylose affinity purification. Both the A312V
I317V and, to a lesser degree, the A312V I317A mutant gave increased yields in the affinity purification compared to wildtype for both the MBP2 and MBP2-CBD derivatives (Table 1).

Dissociation constants for MBP mutants We measured the affinity for maltose and maltotriose for each mutant MBP that retained the ability to enhance the solubility of DHFR. The method we used was ligand-induced fluorescence quenching of tryptophan, essentially as described (Miller et al. 1983). Emission wavelength scans were performed for each mutant, and we discovered that the shape of the curves for the MBP2 derivatives we tested was slightly different from that described in the literature for MBP (Fig. S2). For fluorescence quenching with maltose, we chose an emission wavelength of $350 \mathrm{~nm}$. For fluorescence quenching with maltotriose, we used either 330 and/or $350 \mathrm{~nm}$, depending on the shape of the emission spectrum. In cases where both 330 and $350 \mathrm{~nm}$ titrations were done, the results were in 
Table 1 Solubility and yields of double mutants Solubility was calculated as in Fig. 3

\begin{tabular}{llll}
\hline MBP2 derivative & \% MBP2-DHFR soluble & MBP2 yield (mg/L) & MBP2-CBD yield (mg/L) \\
\hline wt & $62 \%$ & $2.3 \pm 0.5$ & $3.5 \pm 0.9$ \\
A312V I317V & $67 \%$ & $31 \pm 4.8$ & $44 \pm 3.1$ \\
A312V I317A & $74 \%$ & $16 \pm 3.9$ & $22 \pm 4.1$ \\
A312V Q325R & $35 \%$ & ND & ND \\
A312V Q325K & $35 \%$ & ND & ND \\
\hline
\end{tabular}

For solubility, the value for wt is the average of 19 experiments, and the values for the mutants are from two to three experiments. For yields, the value for wt is from 19 experiments, and the values for the mutants are the average of six experiments; errors indicate the $95 \%$ confidence interval $N D$ not determined

good agreement. We determined the dissociation constants using curve-fitting software with the standard equilibrium equation as the model. The results are given in Table 2. In most cases, those mutants that show an increased yield in the affinity purification also show a decrease in the $\mathrm{Kd}$. The exceptions are F67L, where the Kds for both maltose and maltotriose are increased relative to MBP2, and V343M, where the $\mathrm{Kd}$ for maltotriose is increased. We presume that the $\mathrm{Kd}$ for maltotriose is more relevant, since the amylose column is derivatized with maltodextrins of chain length greater than three.

\section{Discussion}

Using random mutagenesis of the sequence encoding the MBP2 protein, we were able to find mutations in MBP2 that enhance its yield in an affinity purification. Nineteen out of the 4,000 mutants screened, or about $0.5 \%$, showed an increase in yield in a microscale amylose resin affinity purification. The distribution of the mutations was nonrandom, with six mutations clustered between residues 64 and 115 in the Nterminal half of the protein, and the remaining 13 mutations clustered between residues 303 and 367 in the C-terminal half. We obtained multiple isolates where the same residue was mutated, suggesting that we had isolated mutations in most of the residues that could be obtained using this method of mutagenesis and screening.

Models for altered affinity There are a number of models that could explain the increased binding of our MBP mutants. The previous work has focused on the fact that the open conformation of MBP is stabilized by a large area of contact between the two domains behind the hinge (Marvin and Hellinga 2001a; Telmer and Shilton 2003). In the maltosebound closed form, the corresponding areas of this interface are solvent-exposed. Alterations of MBP that disturb the interface alter the equilibrium toward the closed form, and affinity for maltodextrins is increased, since the energetic penalty for separating the interface is reduced or eliminated.
One could also imagine that alterations that change the geometry of the hinge loops could alter the equilibrium between the open and closed conformations in a similar manner. Another way to affect ligand binding might be to alter the binding site, by mutating residues that form contacts to the ligand, or indirectly, by making nearby changes that move those residues slightly. Finally, MBP makes extensive contacts to the MalFGK transporter as it docks and releases its ligand, especially between domain I of MBP and the P2 loop of MalF (Oldham et al. 2007). It is possible that these contacts induce a conformational change in MBP to reduce its affinity for the ligand, and mutations in MBP that disfavor this hypothetical shift might increase its affinity.

Mutations that affect the interface behind the hinge Several of our mutations, located between residues 300 and 330, can be rationalized by reference to the previous sitedirected work and fit the model that biasing the conformational equilibrium towards the closed form increases MBP's affinity for maltodextrins. This region includes residues that were modified by Hellinga's lab (I329) and Shilton's lab (M321 and Q325). In fact, we obtained mutations in the latter two residues in our screen. These mutations interfere with the packing of the interface between the two domains

Table 2 Dissociation constants of a subset of the mutant proteins

\begin{tabular}{lll}
\hline & Kd maltose & Kd maltotriose \\
\hline MBP2 & $1.49 \pm .01$ & $0.38 \pm .02$ \\
F67L & $7.00 \pm .7$ & $1.40 \pm .2$ \\
A312V & $0.23 \pm .02$ & $0.11 \pm .01$ \\
I317V & $0.60 \pm .08$ & $0.20 \pm .03$ \\
Q325R & $0.52 \pm .05$ & $0.31 \pm .02$ \\
V343M & $0.92 \pm .06$ & $0.54 \pm .05$ \\
A312V I317V & $0.16 \pm .02$ & $0.03 \pm .01$ \\
A312V I317A & $0.86 \pm .06$ & $0.19 \pm .03$ \\
\hline
\end{tabular}

Values are calculated as described in "Materials and methods"; error is calculated from the curve-fitting software, and is not an experimental error 
that forms when MBP is in the open conformation. We predict that our mutation in A318 also falls in this category, and probably T93A, V302A, V302L, Y307C, N323Y, and N332I as well (Fig. 4). The T93 side chain is not part of the interface itself but rather is on the opposite side of helix 5 from F92, which makes van der Waals contacts with domain II in the open conformation. Likewise, the A302, Y307, N323 side chains are on the opposite sides of helix 13 and 14 that form part of the domain II surface of the interface. The K83R mutation does not offer an analogous rationale, but the $\mathrm{K} 83$ side chain undergoes an extensive remodeling during the open to closed transition, and it is possible that the arginine substitution has effects on the position of helix 5 as well. The side chain of N332 forms a link between the two domains by hydrogen bonding to the backbone carbonyl of A96 in the closed conformation and flexes with domain II as the conformation opens. While not directly part of the interface that forms as the conformation shifts to the open form, this hydrogen bond provides a unique way for domains I and II to communicate independent of the hinge regions, by linking the hinge motion to an alteration of the conformation of the loop between helices 14 and 15. It might be possible to test whether these mutations affect the equilibrium between the open and closed forms. NMR experiments using paramagnetic relaxation enhancement (PRE) have shown that in the absence of maltose, MBP exists as a rapidly exchanging mixture of $\sim 95 \%$ open and $\sim 5 \%$ closed form (Tang et al. 2007). Using this technique on the mutant MBPs would allow one to measure the equilibrium between the open and closed forms directly.

Mutations that affect the hinge Several of our mutations are located in or directly adjacent to two of the hinge regions between domains I and II. The mutations V110I and S114L are in or near hinge region 1 (residues 110-113), and $\mathrm{A} 312 \mathrm{~V}$ and $1317 \mathrm{~V}$ are in or near hinge region 3 (residues 311-315). These mutations could also indirectly affect the packing of the interface behind the hinge, or they could affect the conformation of the hinge directly and thus alter the equilibrium between the open and closed conformations. The $\mathrm{A} 312 \mathrm{~V}$ and $1317 \mathrm{~V}$ mutations in particular suggest the latter possibility, as the A312 side chain is solvent exposed in the open conformation but rotates inward and forms van der Waals contacts with I317 in the closed conformation (Fig. 5).
Fig. 4 Residues where mutations may affect packing behind the hinge. a Cartoon of MBP showing residues where mutations were obtained. Colors as in Fig. 2, except labeled residues in red. b Surface representation of MBP in the closed conformation; colors as in (a). c Surface representation of MBP in the open conformation; colors as in (a)

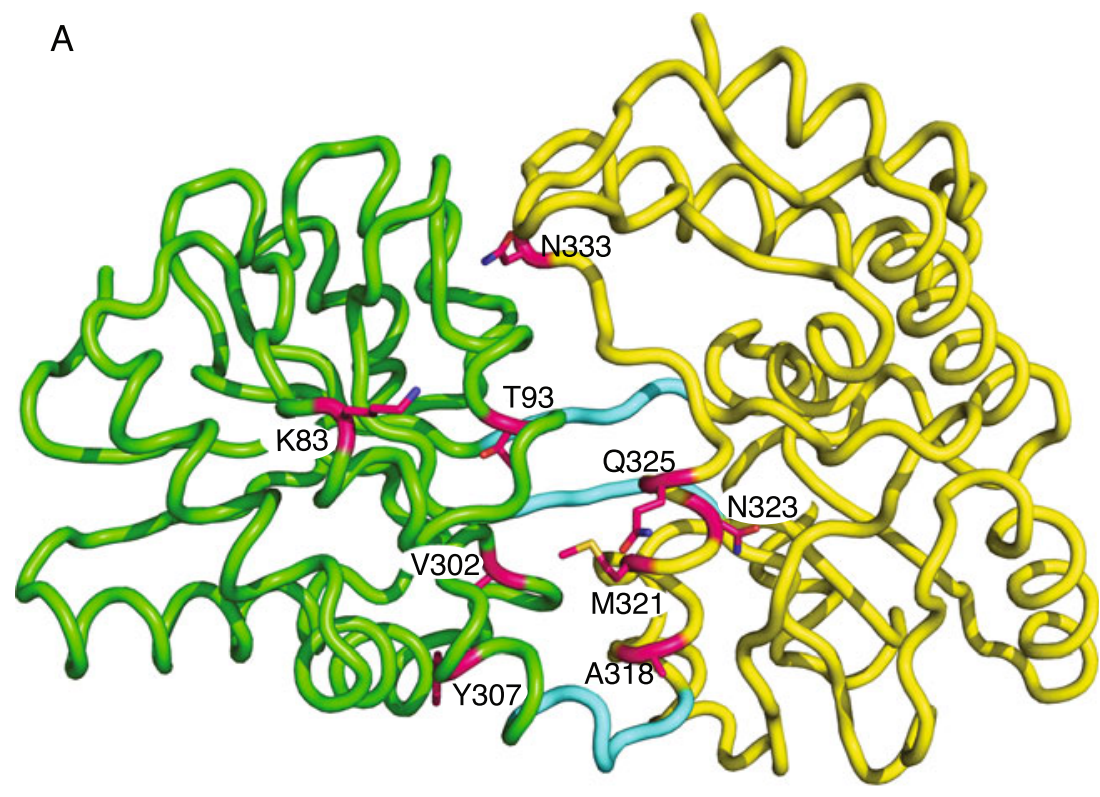

B

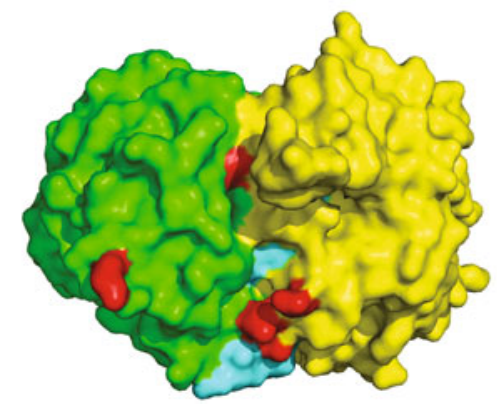

C

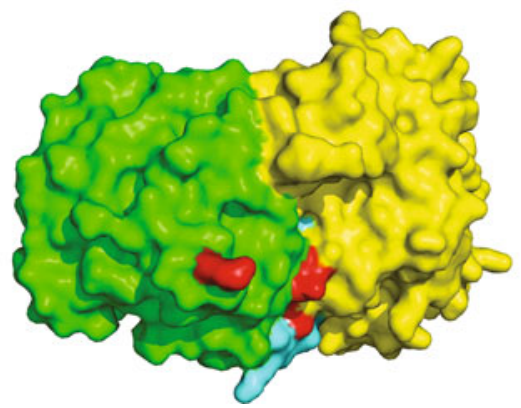



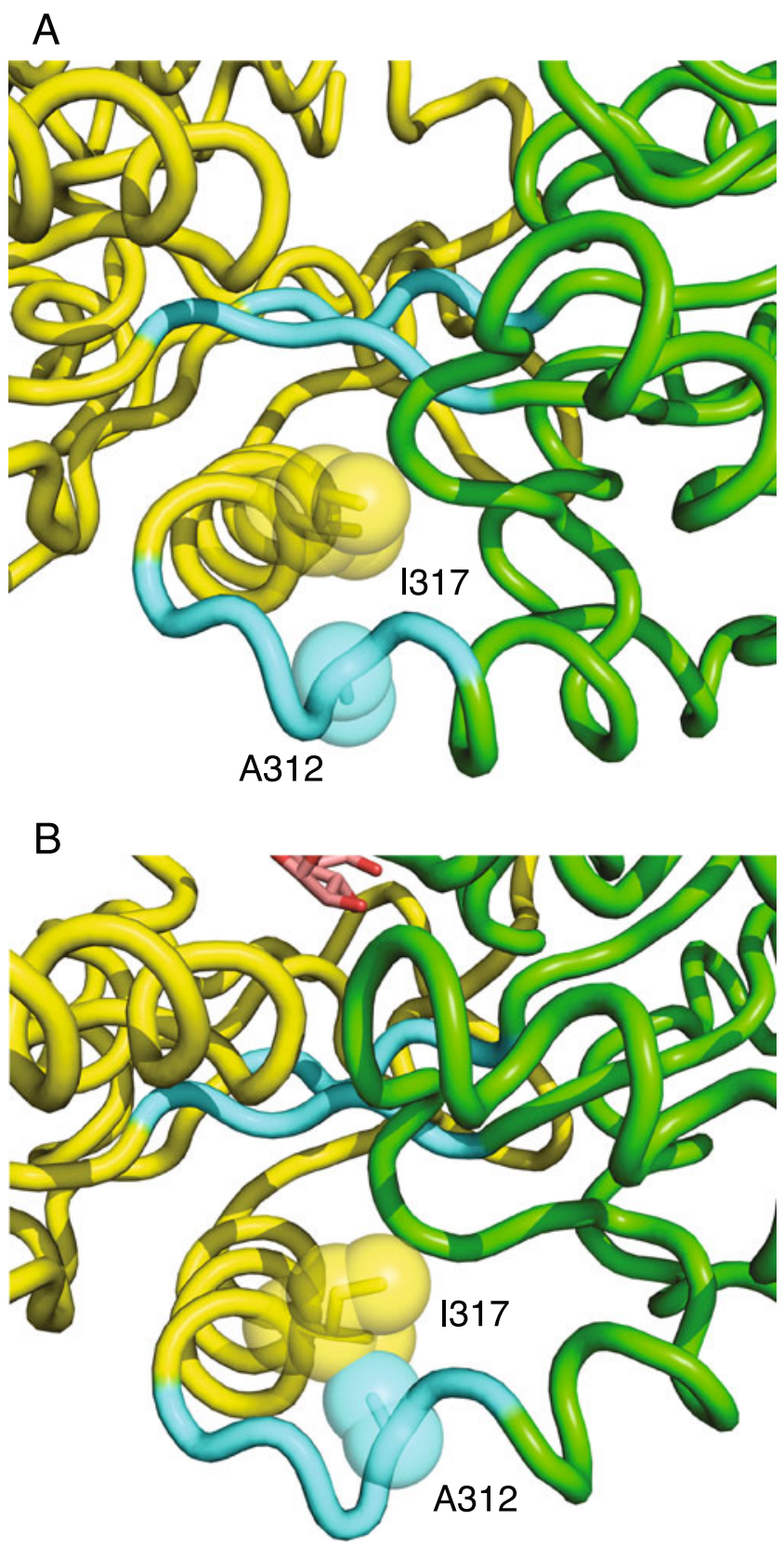

Fig. 5 Position of A312 and I317. a Open (unliganded) conformation, PDB 1JW4 (Duan and Quiocho 2002). b Closed (maltose bound) conformation

Again, NMR PRE experiments would allow one to assess directly the effect of the mutations on the conformational equilibrium.

Mutations away from the interface and hinge The A63V, $\mathrm{F} 67 \mathrm{~L}, \mathrm{~V} 343 \mathrm{M}$, and $\Delta \mathrm{T} 2628$ mutations are located farther from the hinges and the interface behind the hinges than the other mutations, suggesting a different mode of action. The $\mathrm{A} 63 \mathrm{~V}, \mathrm{~F} 67 \mathrm{~L}$, and $\mathrm{V} 343 \mathrm{M}$ mutations are adjacent to residues that form hydrogen bonds to the bound maltose, making it possible that they affect the binding pocket
(Fig. 6). A63 is adjacent to W62, which forms a hydrogen bond to the bound maltose. F67 is on the face of helix 3 opposite to D65 and R66, which both also form hydrogen bonds to maltose. V343 forms van der Waals contacts with P154, which is adjacent to E153 and on the opposite face of helix 7 from Y155. E153 forms a hydrogen bond to maltose and the ring of Y155 stacks with the bound sugar. Paradoxically, the A63V and V343M mutations cause MBP2 to have a lower affinity for maltotriose, at least under the conditions used to measure affinity in this study. It is possible that these mutations have some effect on the kinetics of binding, for example, disproportionately decreasing the off rate of the ligand. However, we performed the Kd measurements under low ionic strength conditions (for comparison to values in the literature), as opposed to the moderate ionic strength we used in the affinity purification. Interestingly, the V343M mutation lies inside a subdomain consisting of residues 165 to183 and 334 to 369 , which has been identified as the part of MBP that varies in position when binding maltose vs. longer maltodextrins (Quiocho et al. 1997). Measuring Kds under a variety of
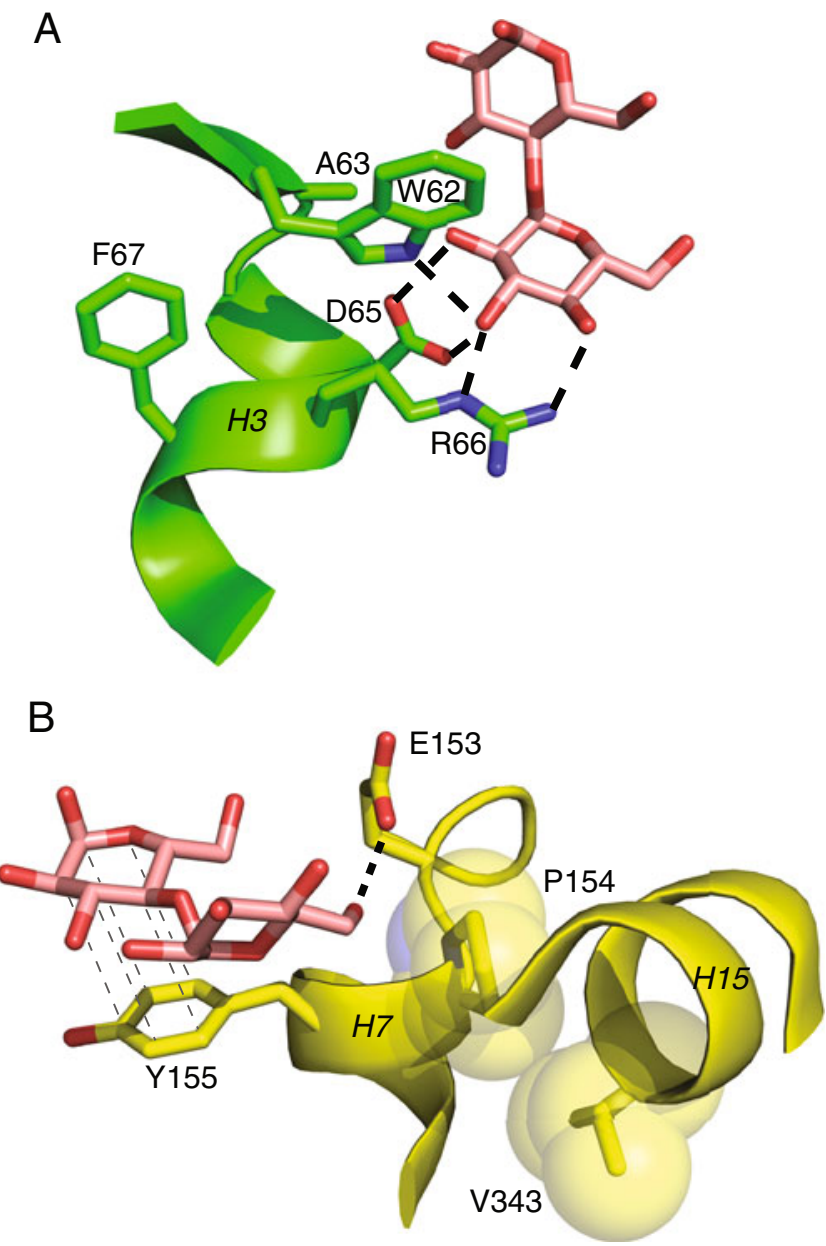

Fig. 6 Location of mutations near the maltose binding site. Colors are as in Fig. 3, with hydrogen bonds indicated as heavy dotted lines, and light dotted lines indicating stacking interactions. a A63 and F67. b V343 
conditions and increasing the number of forms of maltodextrin tested might clarify the effects of these mutations. The $\Delta \mathrm{T} 2628$ mutation is a frameshift mutation that changes all the residues encoded by the synthetic linker following the portion of the malE gene used in this construct. Because of the large number of substitutions caused by this mutation, it will require quite a bit of further study to determine which of these substitutions affect the yield and solubility of MBP2 fusions.

Effects on solubility enhancement Most of the mutations we obtained reduce the ability of MBP2 to enhance the solubility of an MBP2-DHFR fusion protein. From the wide variety of mutations that have this behavior, it seems that this property of MBP2 is somewhat sensitive to alteration. These mutations did not cause MBP2 itself to become insoluble, as the mutant MBP2s had to have reasonable solubility to pass the screen for increased yield in the affinity purification. Fox et al. identified substitutions of glutamate for W232, Y242, and I317 as defective in enhancing the solubility of three aggregation-prone proteins fused to MBP (Fox et al. 2001). These residues are all located at one end of the maltodextrin-binding cleft, potentially defining a site involved in enhancing solubility. However, the mutations in these residues all showed reduced stability as well, which led these researchers to conclude that it could also be some global defect in the folding of MBP that reduced the solubility enhancement. Our widely scattered mutations with the same property of reduced solubility enhancement only confuse the issue further, since they do not fit the model of a site on the surface of MBP that is important for this property. In fact, unlike the I317E mutation of Fox et al., our substitutions of valine and alanine for I317 did not reduce solubility enhancement. We think it is more likely that some of the mutations create disruptions of the folding pathway or possibly in the form or lifetime of a folding intermediate, which also disrupt the interaction between MBP and the protein to which it is fused.

MBP mutants as fusion tags Mutations that increase the yield from the amylose affinity purification and retain the ability of wild-type MBP to enhance the solubility of aggregation-prone proteins have obvious utility as potential fusion tags. Five of the 19 mutations retained the solubility enhancement seen with the MBP2-DHFR fusion. Three of these, A312V, I317V, and Q325R, have higher affinity for maltodextrins, most likely caused by shifting the equilibrium of MBP towards the closed conformation as discussed above. The reason for the increased yield of MBP2 fusions containing F67L and V343M is more obscure, although the F67 and V343 residues are located adjacent to residues that form bonds to the bound maltodextrin. The double mutants A312V I317V, and A312V I317A show an even higher affinity while maintaining solubility enhancement. The increased yield of these MBP derivatives when purified on immobilized amylose translates to an increase in yield of at least one fusion protein, the MBPCBD fusion. But it is not clear that all MBP fusions that fail to bind to amylose do so in the same way. One can imagine that one mode of failure could be an interaction between MBP and the fused protein that alters the conformation of MBP. In this case, alterations that bias MBP towards the closed form may improve the binding of the fusion. However, another mode of failure might be misfolding of the fused protein, leading to a nonspecific interaction with the hydrophobic binding cleft of MBP that blocks access to the binding site. In this case, higher binding affinity might not lead to improved binding of the fusion protein. Future work will show how much these mutant MBPs with increased affinity will improve the success rate for purifying fusion proteins by amylose affinity chromatography.

Acknowledgements We thank Ming $\mathrm{Xu}$ and David Waugh for providing plasmids and Jim Samuelson, Claude Maina, and especially Lise Raleigh for helpful comments on the manuscript.

Open Access This article is distributed under the terms of the Creative Commons Attribution Noncommercial License which permits any noncommercial use, distribution, and reproduction in any medium, provided the original author(s) and source are credited.

\section{References}

Abramoff MD, Magelhaes P, Ram SJ (2004) Image processing with ImageJ. Biophoton int 11:36-42

Aslanidis C, de Jong PJ (1990) Ligation-independent cloning of PCR products (LIC-PCR). Nucleic Acids Res 18:6069-6074

Duan X, Quiocho FA (2002) Structural evidence for a dominant role of nonpolar interactions in the binding of a transport/chemosensory receptor to its highly polar ligands. Biochemistry 41:706-712

Fox JD, Kapust RB, Waugh DS (2001) Single amino acid substitutions on the surface of Escherichia maltose-binding protein can have a profound impact on fusion proteins. Protein Sci 10:622-630

Friedhoff P, Gimadutdinow O, Ruter T, Wende W, Urbanke C, Thole H, Pingoud A (1994) A procedure for renaturation and purification of the extracellular Serratia marcescens nuclease from genetically engineered Escherichia coli. Protein Expr Purif 5:37-43. doi:10.1006/prep.1994.1005

Fromant M, Blanquet S, Plateau P (1995) Direct random mutagenesis of gene-sized DNA fragments using polymerase chain reaction. Anal Biochem 224:347-353

Guan C, Kumar S (2005) A single catalytic domain of the junctionresolving enzyme $\mathrm{T} 7$ endonuclease $\mathrm{I}$ is a non-specific nicking endonuclease. Nucleic Acids Res 33:6225-6234

Kapust RB, Waugh DS (1999) Escherichia coli maltose-binding protein is uncommonly effective at promoting the solubility of polypeptides to which it is fused. Protein Sci 8:1668-1674

Marvin JS, Hellinga HW (2001a) Conversion of a maltose receptor into a zinc biosensor by computational design. Proc Natl Acad Sci U S A 98:4955-4960

Marvin JS, Hellinga HW (2001b) Manipulation of ligand binding affinity by exploitation of conformational coupling. Nat Struct Biol 8:795-798 
Miller DM 3rd, Olson JS, Pflugrath JW, Quiocho FA (1983) Rates of ligand binding to periplasmic proteins involved in bacterial transport and chemotaxis. J Biol Chem 258:13665-13672

Nallamsetty S, Waugh DS (2007) Mutations that alter the equilibrium between open and closed conformations of Escherichia coli maltose-binding protein impede its ability to enhance the solubility of passenger proteins. Biochem Biophys Res Commun 364:639-644

Oldham ML, Khare D, Quiocho FA, Davidson AL, Chen J (2007) Crystal structure of a catalytic intermediate of the maltose transporter. Nature 450:515-521. doi:10.1038/nature06264

Quiocho FA, Spurlino JC, Rodseth LE (1997) Extensive features of tight oligosaccharide binding revealed in high-resolution structures of the maltodextrin transport/chemosensory receptor. Structure 5:997-1015
Sharff AJ, Rodseth LE, Spurlino JC, Quiocho FA (1992) Crystallographic evidence of a large ligand-induced hinge-twist motion between the two domains of the maltodextrin binding protein involved in active transport and chemotaxis. Biochemistry 31:10657-10663

Spurlino JC, Lu GY, Quiocho FA (1991) The 2.3-A resolution structure of the maltose- or maltodextrin-binding protein, a primary receptor of bacterial active transport and chemotaxis. $\mathrm{J}$ Biol Chem 266:5202-5219

Tang C, Schwieters CD, Clore GM (2007) Open-to-closed transition in apo maltose-binding protein observed by paramagnetic NMR. Nature 449:1078-1082. doi:10.1038/nature06232

Telmer PG, Shilton BH (2003) Insights into the conformational equilibria of maltose-binding protein by analysis of high affinity mutants. J Biol Chem 278:34555-34567 